ARTIGO

Recebido em: $30 / 08 / 2013$

Aceito em: 05/11/2013
Encontros Bibli: revista eletrônica de biblioteconomia e ciência da informação, v. 18, n. 38, p. 1-18, set./dez., 2013. ISSN 1518-2924. DOI: 10.5007/1518-2924.2013v18n38p1

\section{Inteligencia Organizacional: conceptos, modelos y metodologias}

\section{Organizational intelligence: concepts, models and methodologies}

Adela HABER-VEJA ${ }^{1}$ Anays MÁS-BASNUEVO²

\title{
RESUMEN
}

Se realiza un análisis de los conceptos, modelos y metodologías de la Inteligencia desde la perspectiva organizacional, haciendo énfasis en la Inteligencia Organizacional (IO). Se visualizan las principales semejanzas y diferencias, a partir del análisis de las principales definiciones de la perspectiva organizacional de la inteligencia. Se caracterizan los modelos consultados para el desarrollo de la IO, a partir de las investigaciones y aplicación en la práctica para su introducción y desarrollo; y se relacionan algunas limitaciones de las metodologías expuestas, a partir de un breve análisis de las mismas. PALABRAS CLAVE: Inteligencia Organizacional. Inteligencia Empresarial. Inteligencia Competitiva.

\begin{abstract}
It is carried out an analysis of the concepts, models and methodologies of Intelligence from an organizational perspective, doing emphasis on Organizational Intelligence. It visualized main similarities and differences, based on the analysis of the main definitions of the organizational perspective of intelligence. Consulted Models to development of Organizational Intelligence are characterized, starting from research and practical application for their introduction and development, and relate some limitations of the discussed methodologies, from an brief analysis of the same.
\end{abstract}

KEYWORDS: Organizational Intelligence. Business Intelligence. Competitive Intelligence.

\section{MÉTODOS}

Analítico-sintético: En la consulta, extracción y recopilación de la información relacionada con la inteligencia en el contexto organizacional. Histórico-lógico: En el estudio y análisis de los antecedentes y desarrollo del proceso de gestión de la Inteligencia Organizacional.

v. 18, n. $38,2013$. p. $1-18$ ISSN 1518-2924

${ }^{1}$ Delegación Provincial del CITMA - La Habana - Cuba - $\underline{\text { adela@delegcha.cu }}$
${ }^{2}$ Consultoría Biomundi, IDICT - La Habana - Cuba - $\underline{\text { anays@biomundi.inf.cu }}$ 


\section{INTRODUCCION}

La inteligencia en el contexto de las organizaciones es tratada, de acuerdo con la dimensión y el contexto de su práctica, con diferentes denominaciones (empresarial, para los negocios, sobre los competidores, monitoreo del medio, económica, corporativa o competitiva), que aunque la reconocen como capacidad o cualidad de la organización, está enfocada al aumento de sus rendimientos económicos y a su posicionamiento en el mercado, comprometiendo la evaluación de los resultados de su implantación, con el cumplimiento de su objetivo.

“En los años 90 se introduce en el campo empresarial el concepto de inteligencia con un carácter más integral en relación a los métodos tradicionales de seguimiento del entorno" (1 p.2) como la vigilancia pasiva (scanning) y la vigilancia activa (monitoring). En el contexto empresarial, la inteligencia empresarial (IE) es tratada como equivalente de la inteligencia organizacional, los anglosajones la denominan Inteligencia para los negocios (business intelligence), Inteligencia sobre los Competidores (competitor intelligence) y Monitoreo del medio (environmental scanning); los franceses como Inteligencia Económica (Veille Economique); y los españoles utilizan el término Inteligencia Corporativa como sinónimo de Inteligencia Competitiva (IC).

Muchos autores han conceptualizado la IC con diferentes denominaciones, de acuerdo con la dimensión y el contexto de su práctica, sin que haya un consenso al respecto. La IC ha sido definida como proceso (2); (3); (4); (5); (6); (7); (8); (9); (10); (11); (12), como sistema (13), y como proceso, función, producto o su fusión (14). Las etapas descritas por estos autores para la gestión de la IC están relacionadas con el ciclo de la inteligencia y contemplan la búsqueda, obtención, análisis, interpretación y diseminación de información relevante, de forma ética y legal, sobre el entorno de la organización para la toma de decisiones y para la orientación estratégica (3); (4); (5); (6); (7); (8); (10); (11); (15). Mientras Clerc (9) incluye la protección de la información; Canongia (16) la planificación y la retroalimentación; y Miller (17) la identificación de los principales responsables por las decisiones y sus necesidades en cuestión de inteligencia.

Orozco (18) explica que la diferencia entre inteligencia empresarial e inteligencia competitiva radica en que la segunda se refiere solo al ambiente de los competidores y sus capacidades, vulnerabilidad e intenciones; es una parte de la primera que tiene un enfoque más amplio y abarca todos los aspectos del trabajo de la organización. La 
inteligencia empresarial es "la capacidad de reunir, analizar y diseminar datos, lo que permite obtener, de manera sistemática y organizada, información relevante sobre el ambiente externo y las condiciones internas de la organización, para la toma de decisiones y la orientación estratégica" (19 p. 6) y su ejercicio comprende los pasos del ciclo de la inteligencia: "la identificación del problema que se quiere resolver; la definición de las fuentes de información; la búsqueda de datos, su evaluación y validación; y la conversión en información mediante un proceso de organización, homogenización y contextualización; el análisis de la información; la interpretación de los resultados; y la confección del informe y su diseminación" (19 p. 10).

Este enfoque de la inteligencia aun cuando le atribuye a la inteligencia empresarial un alcance más general e integrador que la vigilancia y la inteligencia competitiva, reconociendo la inteligencia como capacidad o cualidad de la organización; las etapas para su gestión, que describen el cómo, solo comprenden los procesos informacionales de agregación de valor concernientes a la gestión del ciclo de vida de la información relacionada con el mundo del conocimiento del modelo de Vizcaya (20).

Se coincide con Orozco (21), Núñez Paula (22) y Más (23) cuando plantean que el concepto de IO debe ser considerado como el más genérico al interpretar la capacidad de la inteligencia en el contexto de cualquier tipo de organizaciones, como se muestra en la Figura 1.
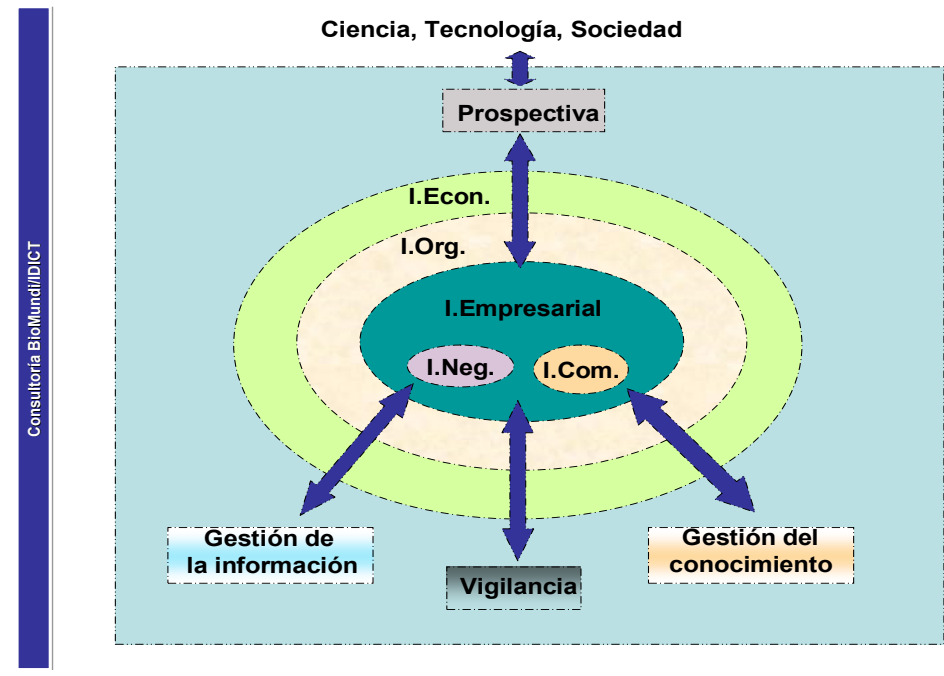

Figura 1. Relación de la IE con otras herramientas útiles para el desarrollo de la ciencia, la tecnología y la sociedad

Fuente: 10mo. Taller Brasileño de Inteligencia Competitiva y Gestión del Conocimiento (24)

Leyenda: $\quad$ I.Empresarial - inteligencia empresarial

I.Neg. $\quad-$ inteligencia de negocios

I.Com - inteligencia competitiva

I.Org. $\quad-$ inteligencia organizacional

I.Econ. - inteligencia económica 
En la Figura 1 no solo se muestra el lugar que ocupa la IO dentro de las acepciones de inteligencia que se tratan en este trabajo, sino también que se describe el marco conceptual bajo el cual la Consultoría BioMundi/IDICT presta servicios de IE, donde:

- la gestión de la información y del conocimiento, y la vigilancia son imprescindibles para la IE;

- la inteligencia competitiva y de negocios son subconjuntos de la empresarial. De esta manera, la IE puede necesitar estudios de la competencia o de las tecnologías para la gestión de la información interna del negocio, pero la relación no es bidireccional: iniciativas de inteligencia de negocio o de inteligencia competitiva no significan que la organización use la IE;

- para la inteligencia organizacional pueden usarse productos y servicios de la IE, pero ella es mucho más, porque está dirigida a la creación de capacidades internas y el aprendizaje continuo de los espacios de intercambio dentro de las organizaciones;

- la inteligencia económica es "la combinación de todas las medidas coordinadas de colección, procesamiento, distribución y protección de información valiosa para los sectores económicos y que se puede obtener por medios legales. Su objetivo es ofrecer a los decidores en las empresas o en el gobierno el conocimiento necesario para comprender el ambiente y ajustar las estrategias individuales o colectivas de acuerdo con el conocimiento obtenido" (9); y

- la prospectiva se beneficia de los resultados que se obtienen con una efectiva gestión de la información y del conocimiento, y el valor agregado que genera cualquier tipo de inteligencia, un conjunto de algunas de ellas o todas.

Obsérvese que no hay suscripción al apellido tecnológico de la vigilancia y la prospectiva, porque eso reduce el rango de aplicación de estas herramientas.

\section{INTELIGENCIA ORGANIZACIONAL (IO)}

Matsuda (25) plantea que la IO puede ser vista como un proceso o como un producto. Considera que la inteligencia está compuesta por varios procesos: percepción, almacenamiento, aprendizaje, comunicación y decisión. Según Choo (26) la IO fue 
definida por Wilensky en términos de reunir, procesar, interpretar y comunicar la información necesaria en los procesos de toma de decisiones; y con un enfoque similar Haeckel y Nolan la conceptualizan como la habilidad para capturar, compartir y extraer significado de las señales del mercado.

Estos conceptos enfatizan en:

a) el reconocimiento del valor de la información del ambiente externo de la organización, para la definición de su accionar a partir de la gestión del ciclo de vida de la información, que abarca las perspectivas de los sistemas de información y el contexto de su repercusión, propuesto por Vizcaya (20);

b) los procesos informacionales de agregación de valor; y

c) el proceso de toma de decisiones.

Sin embargo, no reconocen la inteligencia como capacidad o cualidad de la organización, ni su formación y desarrollo a nivel individual, grupal y organizacional, a partir de la comunicación interactiva que se produce en las relaciones que se establecen entre estos niveles, en la solución de problemas o en la realización de acciones, que propicien la creación y transferencia del conocimiento.

Con una visión más amplia de estos enfoques, Oberchulste (27) entiende la IO como la capacidad de afrontar nuevas exigencias o nuevos cometidos; Halal como la "capacidad de una organización para crear conocimiento y utilizarlo para adaptarse estratégicamente a su entorno" (28). Para McMaster es "la capacidad de detectar, de tener sentido y de tomar la acción, combinada con el conocimiento que es disponible y listo para utilizarse" (29). En estas definiciones se reconoce la inteligencia como capacidad que le permite a la organización adaptarse al ambiente externo; la creación y uso del conocimiento como procesos que favorecen la formación y desarrollo de la IO y la toma de decisiones estratégicas.

Choo profundiza en estas definiciones y define la IO como "la propiedad que emerge de la red de procesos del uso de información a través de la cual la organización construye significados compartidos sobre sus acciones e identidad; descubre, comparte y aplica nuevo conocimiento, e inicia patrones de acción a través de la búsqueda, evaluación y selección de opciones" (26 p. xviii).

Para Núñez Paula la IO es derivada del significado de la inteligencia social e “implicaría la utilización efectiva de la información del entorno de la organización y de 
todas las operaciones internas, del conocimiento -en su sentido más abarcadorexistente fuera y dentro de la organización, en función de la innovación o el mejoramiento continuo, el aprovechamiento de oportunidades, la creación de nuevo conocimiento y valor, la formación y el bienestar de las personas dentro de la organización, así como de los clientes y otras partes interesadas y de la sociedad en su conjunto" (22 p. 4). North y Pöschl (30) entienden la IO como la capacidad de resolución de problemas y de realizar nuevas tareas, dependiendo de la eficacia y rapidez con que los diferentes componentes de estos procesos operan y cómo aquellos se hayan relacionados entre sí funcionalmente. La IO resulta de la cooperación entre los portadores de conocimiento.

Más define la IO como "capacidad y necesidad de los individuos de la organización, y de esta en su totalidad, de desarrollar actividades en función del proceso sistematizado y controlado de planificar, recolectar, analizar, producir, proteger, diseminar, conservar y evaluar información y conocimientos normalizados y relevantes sobre el medioambiente en que se desenvuelve la organización de manera que la toma de decisiones sea más efectiva" (23 p. 34).

Choo, Núñez Paula, North y Pöschl, y Más, coinciden con las propuestas de valor agregado de la información hasta llegar a la inteligencia de Páez Urdaneta (31) y de Taylor (32), y con el basamento en el paradigma sociocultural del aprendizaje expuesto por Vigotsky.

El análisis de las principales definiciones de la perspectiva organizacional de la inteligencia, atendiendo a su alcance, etapas y a los aspectos en que enfatizan, permite visualizar las principales semejanzas y diferencias entre ellas.

Semejanzas:

- Reconocimiento de la información y el conocimiento como recursos y la necesidad de su efectiva gestión por su importancia para la toma de decisiones y la orientación estratégica.

- Asunción de la concepción cognitivista o de procesamiento de la información para la formación y desarrollo de la inteligencia.

- Visualización de los procesos informacionales de agregación de valor a partir de las etapas del ciclo de la información y su gestión, para fomentar la innovación y generar valor. 
- Relación de la inteligencia con el desempeño organizacional de acuerdo con su alcance, contexto y fines.

Diferencias:

- La IC y la IE constituyen herramientas gerenciales; la IO es además, un instrumento cultural, una forma de hacer a todos los niveles de la organización.

- Al reconocer la IC y la IE como función de la organización, los productos de información de alto valor agregado obtenidos como resultado de los servicios de inteligencia, pueden ser generados en la organización o contratados a una organización especializada en estos servicios. La IO es una capacidad general inherente a la organización, solo sería posible contratar la metodología para su diagnóstico, implantación y evaluación; y como parte de este proceso, subcontratar servicios relacionados con las condiciones que favorecen su formación y desarrollo.

- Los resultados de la IC y de la IE tienen un uso inmediato y un carácter temporal; los de la IO forman parte de la cultura organizacional, se manifiestan en patrones de comportamientos que producen sentimientos compartidos a todos los niveles de la organización por alcanzar resultados más efectivos en el cumplimiento de sus metas, a partir de la necesidad de usar y compartir la información y el conocimiento.

- La IO incluye la combinación del paradigma cognitivo con el paradigma sociocultural para desarrollar la capacidad de aprendizaje continuo a todos los niveles organizacionales.

El análisis de estas diferencias, además de las enunciadas por Orozco (21), Núñez Paula (22) y Más (23), reafirman la consideración del concepto de IO como el más genérico al interpretarlo en cualquier contexto organizacional.

\section{MODELOS DE IO}

Más (23) analiza 15 modelos de Inteligencia teniendo en cuenta las etapas y/o funciones que comprenden, así como el aspecto de la IO en que enfatizan y concluye que el $66,7 \%$ no explicita la formación de conocimientos o inteligencias a niveles individuales, grupales u organizacionales, a partir de la capacidad o cualidad de la 
organización para combinar sentimientos con conocimiento y acción y formar ciclos continuos de interpretación, innovación e iniciativa; lo que los enmarca desde el punto de vista psicológico, en el paradigma cognitivo del procesamiento humano de la información.

Otros modelos consultados para el desarrollo de la IO, a partir de las investigaciones y aplicación en la práctica, Halal (28), Choo (26), North y Pöschl (30) y Más (23), enfatizan en aspectos que constituyen condiciones necesarias para introducir la IO y tienen en común el reconocimiento de la necesidad de desarrollar el proceso de IO, resultante de la relación dinámica y sistémica de los procesos informacionales de agregación de valor y su gestión:

a) por los beneficios que reporta la formación y desarrollo de la inteligencia a nivel individual, grupal y organizacional en la solución efectiva de problemas y en el incremento del capital intelectual de la organización;

b) a través de la interacción de los individuos con otros (grupos) internos y del entorno;

c) sobre la base del uso y el intercambio de información de la organización y del contexto;

d) favorecido por una cultura organizacional que fomente el aprendizaje continuo, la creatividad y la innovación;

e) evaluado por los resultados de la organización en su accionar para cumplimentar su estrategia.

Halal (28) y North y Pöschl (30) extrapolan a las organizaciones la medición de la inteligencia humana, a partir de test psicológicos y expresada en términos de Coeficiente de Inteligencia, y establecen conceptualmente variables para determinar y analizar el nivel de inteligencia en las organizaciones como capacidad potencial para obtener resultados más efectivos en su accionar. Enfatizan en el uso óptimo de la información y en la potencialidad de la inteligencia emocional para incrementar el flujo de conocimiento en las relaciones sociales de los grupos de interés para la organización.

Choo establece su modelo a partir de concebir tres formas de uso estratégico de la información (percepción, creación de conocimiento y toma de decisiones) como un proceso social, dinámico y sistémico envuelto en capas de contingencias cognoscitivas, afectivas y situacionales que se sustentan entre sí. Esa acción introduce variaciones en el 
medio ambiente y origina nuevos torrentes de experiencia a los que la organización debe adaptarse, y de este modo comienza otro ciclo" (26 p. 29). La concepción del modelo visualiza el manejo de la información como un ciclo continuo de seis procesos relacionados: identificación de necesidades de información, adquisición de la misma, organización y almacenamiento de ésta, desarrollo de productos y servicios de la información, distribución y uso.

El modelo propuesto por Más en el contexto de una organización sin fines de lucro, incluye la "combinación de las funciones dinámicas de gestión de información y del conocimiento (paradigma cognitivo) con los sentimientos y voluntades de las personas, para desarrollar la capacidad de aprendizaje continuo (paradigma sociocultural)" (23 p. 46).

En la definición, la autora resalta la IO como capacidad y función individual, grupal y organizacional; y la necesidad de gerenciar el proceso de gestión del ciclo ampliado de la información dentro del proceso de gestión del conocimiento sobre las condiciones internas y externas en que se desarrolla la organización, en su interacción como un todo, para desarrollar el aprendizaje continuo, mejorar la efectividad en la toma de decisiones relacionadas con la gerencia y gestión de la ciencia, la tecnología y el medio ambiente, y fomentar la cultura informacional en una Delegación del Ministerio de Ciencia, Tecnología y Medio Ambiente (CITMA).

Mientras en el modelo, dinamiza a los componentes: a) individuos y grupos, b) procesos en los que participan los individuos y grupos, c) métodos y medios que utilizan los individuos y grupos para ejecutar los procesos y relacionarse y d) las relaciones interpersonales e intergrupales dentro de la organización o interorganizacionales, con el ciclo ampliado de la información, que contiene las mismas etapas que enuncia en la definición (planificación, recolección, análisis, producción, protección, diseminación, conservación y evaluación) como acciones del proceso de IO, en cada una de las cuales se contemplan las etapas del ciclo directivo.

Sin embargo, la evaluación de la introducción de la IO, Más la concibe por el uso del producto de información, previamente protegido, compartido y conservado, en la toma de decisiones y en la creación de nuevas capacidades individuales, grupales y organizacionales. 


\section{METODOLOGÍAS DE IO}

Los conceptos y modelos de IO analizados revelan la validación en la práctica de sus propuestas individuales para la introducción y desarrollo de la IO, teniendo en cuenta los contextos específicos en que se desenvuelven los autores que los proponen.

Halal y North y Pöschl proponen como método para la validación de sus modelos en la práctica, la medición del índice o coeficiente de inteligencia en las organizaciones. Halal define siete variables (Sistemas y Tecnologías de Información, Estructura organizacional, Activos del Conocimiento, Procesos Estratégicos, Cultura Organizacional, Relaciones de interesados, y Rendimiento) para realizar la medición y establece una media para ello que normaliza a 100 , por lo que un coeficiente de inteligencia organizacional por encima de 100 indica una organización más inteligente, mientras que una por debajo de esta media indica que la organización es menos inteligente.

North y Pöschl proponen una matriz que relaciona cinco dimensiones (Mercados / Competidores, Clientes / Proveedores, Productos, Procesos, y Empleados) cada una de las cuales debe ser evaluadas con respecto a: Capacidad de respuesta, Capacidad para resolver problemas, Capacidad de aprender / creatividad / innovación, Memoria Organizacional, Inteligencia Emocional; con el objetivo de que la empresa conozca a profundidad tanto sus capacidades y competencias internas como las de los competidores, para evaluar y medir su situación competitiva.

Pinheiro, P. G.; Hernández Mogollón, R. y M. L. Raposo (33) y Guerra, L.; S. Molinillo y G. Bermúdez (234), refieren la validación del modelo de IO de North y Pöschl a partir de las variables identificadas en la matriz propuesta por estos autores para evaluar el efecto de la práctica de la IO en empresas, integrándolo con el modelo de gestión del conocimiento propuesto por Jordan y Jones, en un modelo innovador para analizar la IO en empresas textiles portuguesas considerando dos fases: 1) las actividades de dirección del conocimiento y; 2) las acciones de la empresa. Para evaluar el modelo planteado realizan un análisis cuantitativo de los indicadores definidos utilizando la matriz de inteligencia y la escala de cinco niveles propuesta por North y las variables referidas por Powell y por Rodrigues para analizar el valor creado por la empresa; y aplican diferentes métodos estadísticos. El análisis realizado permite validar la correlación de la IO con el desempeño de las empresas, pues las que tienen una elevada IO obtienen mayor eficacia y eficiencia en sus acciones. 
Estos autores reconocen algunas limitaciones en su validación del modelo, a saber, no considera la evolución del sector y su IO; la técnica estadística utilizada, Partial Least Squares, tiende a sobrevaluar las cargas factoriales y a reducir los valores de los coeficientes estructurales; no se tienen en cuenta: el contexto en que se desempeñan las empresas; la relación entre la creación, transferencia y uso del conocimiento con la estrategia empresarial; la estructura empresarial; las relaciones intra e interempresariales; ni la importancia del reaprendizaje en el sector. La autora identifica otra limitación; no tiene en cuenta la cultura organizacional y su fomento en el proceso de IO.

Guerra, L.; S. Molinillo y G. Bermúdez (34) examinan los efectos de la IO en la capacidad competitiva de la empresa a partir de los aportes del modelo de inteligencia organizacional de North y Pöschl integrado a los aportes de la tipología de Zammuto, para caracterizar y tipificar el perfil en inteligencia y competitividad organizacional del sector empresarial. Al aplicar diferentes técnicas y métodos estadísticos, identifican tres perfiles de empresas o conglomerados según su orientación dimensional: empresas prospectivas, empresas analizadoras y empresas defensivas.

En el análisis de estas metodologías se pueden observar varias desventajas comunes:

- Están dirigidas a organizaciones con fines de lucro, con el objetivo de aumentar sus rendimientos económicos y posicionarse en el mercado, comprometiendo la evaluación de los resultados de la implantación de la IO con el cumplimiento de su objetivo;

- No utilizan un enfoque holístico y sistémico en la concepción y determinación de las condiciones o factores internos y externos que favorecen su aplicación por su influencia en el proceso de dirección, en el uso de la información, toma de decisiones y desarrollo individual, grupal y organizacional;

- Solo tienen en cuenta las necesidades de información para su gestión, sin identificar las necesidades dinámicas de formación y desarrollo de los individuos para usar y producir continuamente información y conocimientos que favorezcan el desarrollo de las capacidades individuales, grupales y organizacionales.

Choo propone su modelo a partir de los resultados de investigaciones realizadas en Teoría de la Organización y la Ciencia de la información y enmarca su validación para 
la implantación de la IO a través del ciclo de manejo de la información y la evaluación dentro del proceso de toma de decisiones, a partir de la selección de opciones derivadas de la creación, uso compartido y aplicación del nuevo conocimiento resultante del uso de la información, para fomentar el crecimiento y la renovación organizacional.

Más (23) ofrece una Guía para la implantación de la Espiral de Inteligencia Organizacional en la Delegación del CITMA de Holguín, conformada por siete lineamientos que contemplan los elementos esenciales para el diagnóstico de los factores influyentes en la organización; y su implantación, a través del ciclo ampliado de la información que vincula sistémicamente procesos, personas, grupos, métodos y medios y relaciones; y la evaluación del impacto social, tecnológico y medio ambiental de su introducción en la Delegación. La evaluación la realizó de forma parcial en la referida Delegación, a través de variables e indicadores de calidad establecidos (gestión de procesos, gestión de recursos humanos, gestión de métodos y medios y gestión de relaciones), los resultados del análisis del método de consulta a expertos, y la investigación-acción, y demuestra que la aplicación del modelo propio de desarrollo continuo logra una mejoría en la gerencia y gestión de ciencia, tecnología y medio ambiente y fomenta la cultura informacional en la mencionada organización.

\section{LIMITACIONES DE LAS METODOLOGÍAS ANALIZADAS}

Un breve análisis de las metodologías expuestas nos permite valorar algunas limitaciones.

1. Compromisos individuales de los autores con la evaluación de sus contextos de actuación.

2. No se definen las etapas, fases, acciones y pasos, y su interrelación sistémica y cíclica, que regulen el diagnóstico, la implantación y la evaluación de la IO en una organización con o sin fines de lucro.

3. No se definen para cada acción las fuentes documentales y no documentales; las técnicas a emplear, para la obtención de la información según las fuentes; ni las salidas esperadas.

4. No se proponen indicadores para evaluar a) los factores que influyen en la implantación de la IO, como condiciones que es necesario crear o redimensionar para ello; b) el impacto de la implantación de la IO en la efectividad del 
cumplimiento de la misión organizacional y en la toma de decisiones; y c) la percepción de los actores sociales con los que interactúa la organización sobre los productos de información de valor agregado elaborados por la misma.

A pesar de estas limitaciones, cada una de las metodologías propuestas es un esfuerzo por facilitar la introducción del proceso de IO en organizaciones con diferentes fines y situadas en disímiles contextos económicos, políticos, científicos, tecnológicos, sociales, culturales, regulatorios y medioambientales, en aras de adaptarse y transformar el medio; aprender, crecer y desarrollarse.

"Lo anterior solo se logra a través de procesos continuos de generación y uso del conocimiento, organizados de tal forma que puedan contribuir a procesos de aprendizaje social. Este es el rasgo central de las Sociedades del Conocimiento... con capacidad para generar conocimiento sobre su realidad y su entorno, y con capacidad para utilizar dicho conocimiento en el proceso de concebir, forjar y construir su futuro. Es decir, de utilizarlo en el proceso permanente de construcción de la sociedad, a través del desarrollo, transformación permanente y consolidación de sus principales instituciones sociales. De esta forma, el conocimiento se convierte no solo en instrumento para explicar y comprender la realidad, sino también en motor de desarrollo y en factor dinamizador del cambio social" (35 p. 19).

En la Sociedad del Conocimiento, cada individuo, grupo y organización, formará y desarrollará su propia capacidad o inteligencia para accionar y generar nuevo conocimiento, a partir de la aplicación del conocimiento disponible que agregue valor a los procesos, productos o servicios que aporta a la sociedad, y que le permita adaptarse dinámicamente a los cambios del entorno; aprender, y progresar.

Las organizaciones que aprenden son asociadas a organizaciones inteligentes; que reconocen a la información y el conocimiento como recursos y sienten la necesidad individual, grupal y organizacional de incrementar su valor a través de su gestión sistemática, integrada y regulada para solucionar, evitar o proponer la solución de problemas efectivamente, y desarrollar sus competencias o inteligencia individuales, grupales y organizacionales, mediante la comunicación que se establece entre estos niveles y a nivel interorganizacional (trabajo en equipo) en la gestión de los procesos organizacionales; marcado por la formación de condiciones culturales, sociales e históricas, que propician el compromiso colectivo para adaptarse y transformar el 
entorno, aprender continuamente, desarrollar permanentemente la creatividad y la innovación; refrendado en la efectividad de los resultados del cumplimiento de las metas de la organización y su impacto en la sociedad.

Todo el análisis anterior demuestra la factibilidad de asumir la definición y el modelo de IO de Más (23) para su introducción en una organización con o sin fines de lucro; y la necesidad de elaborar una Metodología para implantar su modelo y evaluar su impacto en la mejora de la gestión de los procesos para cumplir la misión organizacional y en su cultura informacional.

\section{A MODO DE CONCLUSIONES}

1. El análisis de los conceptos de inteligencia desde la perspectiva organizacional reafirman la consideración del concepto de IO como el más genérico al interpretarlo en cualquier contexto organizacional.

2. Los modelos de IO que se analizaron enfatizan en aspectos que constituyen condiciones necesarias para su introducción y tienen en común el reconocimiento de la necesidad de desarrollar el proceso de IO, como resultado de la relación dinámica y sistémica de los procesos informacionales de agregación de valor y su gestión.

3. Las metodologías analizadas constituyen un esfuerzo por facilitar la introducción del proceso de IO en organizaciones con diferentes fines y situadas en disímiles contextos, en aras de adaptarse y transformar el medio; aprender, crecer y desarrollarse. Sin embargo, presentan como limitaciones principales: los compromisos individuales de los autores con la evaluación de sus contextos de actuación; no se definen las etapas, fases, acciones y pasos, y su interrelación sistémica y cíclica, que regulen el diagnóstico, la implantación y la evaluación de la IO en una organización con o sin fines de lucro; ni las fuentes documentales y no documentales; las técnicas a emplear, para la obtención de la información según las fuentes; ni las salidas esperadas; y no se proponen indicadores para evaluar los factores que influyen en la implantación de la IO y su impacto en el cumplimiento de la misión organizacional.

4. El análisis documental realizado sobre los antecedentes, conceptos, modelos y metodologías de IO, demostró la necesidad de elaborar una Metodología que regule su diagnóstico e implantación, así como la evaluación de su impacto en la mejora de 
los procesos para cumplir con la razón de ser organizacional y de su cultura informacional.

\section{REFERENCIAS BIBLIOGRÁFICAS}

ASHTON, W. and STACEY, G. "Technical intelligence is business: understanding technology threats and opportunities". International Journal of Technology Management. 1995, vol. 10, núm. 1, p. 79-104.

ASHTON, W. B. and KLAVANS, R. Keeping abreast of science and technology: Technical intelligence for business [CD-ROM]. Columbus, OH: Battelle Press, 1997.

ASSOCIAÇÃO BRASILEIRA DOS ANALISTAS DE INTELIGÊNCIA COMPETITIVA-ABRAIC, 2007. [en línea]. Disponible en Web: <http://www.abraic.org.br>. [Consulta: 25 de marzo de 2010].

CANONGIA, C et al. "Convergência da inteligência competitiva com a construção de visão de futuro: proposta metodológica de Sistema de Informação Estratégica (SIE)". DataGramaZero- Revista de Ciência da Informação. Jun. 2001, vol.2, núm. 3. [en línea]. Disponible en Web: <http://www.dgz.org.br>. [Consulta: 23 de enero 2006].

CHAPARRO, F. Conocimiento, aprendizaje y capital social como motor de desarrollo. Ciencias de la Información Brasilia. jan./abr. 2001, vol. 30, núm. 1, p. 19.

CHOO, C. W. La organización inteligente. El empleo de la información para dar significado, crear conocimiento y tomar decisiones. México: OXFORD University Press, 1999, p. 9.

CLERC, Phillip. Economic Intelligence. World Information Report 1997-98. [París]: UNESCO, 1997. p. 304-317.

COTTRILL, 1998. Citado por: ESCORSA, P. and MASPONS, R. De la Vigilancia Tecnológica a la Inteligencia Competitiva. Madrid: Prentice Hall, 2001, 165p.

ESCORSA, P., MASPONS, R., and CRUZ, E. Inteligencia competitiva y transferencia de tecnologías: Reflexiones para el desarrollo Universidad-Empresa. (ES.3.194). Documento electrónico, p.2.

ETTORE, B. "Managing competitive intelligence". Management Review. American Management Association. 1995, p.15-19.

GIBBONS Y PRESCOTT, 1996. Citado por: ESCORSA, P. and MASPONS, R. De la Vigilancia Tecnológica a la Inteligencia Competitiva. Madrid: Prentice Hall, 2001, $165 \mathrm{p}$.

GIBBONS, P.T. "Parallel competitive intelligence processes in organizations". International Journal of Technology, Special Issue on Informal Information Flow Management. 1996, vol. 11, núm. 1, pp. 162-178.

GILAD, B. "What you don't know, can hurt you: formalising competitive intelligence activities". Journal of AGSI, 1992, p.107-116.

GILAD, B. and GILAD, T. The business intelligence system: a new toll for competitive advantage. En [CD-ROM] New York: Amacom.

GUERRA, L., MOLINILLO, S., and BERMÚDEZ, G. Inteligencia organizacional como elemento dinamizador de configuraciones organizativas competitivas del sector empresarial. 2009 [en línea]. Disponible en Web: <http://www.revistaespacios.com/a09v30n03/09300321.html> [Consulta: 22 de abril de 2011]. 
HALAL, W. Organizational intelligence: What is it, and how can managers use it? [en línea] Disponible en Web: <http://www.strategy-business.com/briefs/97413.html>. [Consulta: 25 de junio de 2008].

HEISER, 1994. Citado por: ESCORSA, P. and MASPONS, R. De la Vigilancia Tecnológica a la Inteligencia Competitiva. Madrid: Pearson Education, 2001, 165p.

JAKOBIAK, 1995; Citado por: ESCORSA, P. and MASPONS, R. De la Vigilancia Tecnológica a la Inteligencia Competitiva. Madrid: Pearson Education, 2001, 165p.

MÁS, B. A. "Modelo para la introducción de la inteligencia organizacional en la Delegación del CITMA en Holguín". Tesis para optar por el grado de Doctor en Ciencias de la Información. Universidad de la Habana, Facultad de Comunicación, 2005. p.34.

MÁS, B. A. and OROZCO, S. E. "Formación en inteligencia empresarial: generación y uso del conocimiento". En: 10mo. Taller Brasileño de Inteligencia Competitiva y Gestión del Conocimiento. Brasilia, Brasil, 2012.

MATSUDA (1992). Citado por: Pinheiro, P. G.; Hernández Mogollón, R. y M. L. Raposo (2007). La inteligencia organizativa de las empresas del sector textil portugués. [en línea]. Disponible en: http:// www.dialnet.unirioja.es. Consultado: 03/08/2009.

MCMASTER, M. Organizacional Intelligence. [en línea] Disponible en Web: <http://www.parshift.com/speakers/speak011.html>. [Consulta: 20 de diciembre de 2005].

MILLER, J. P. 0 milênio da inteligência competitiva. Porto Alegre: Bookman, 2002.

NORTH, K. and PÖSCHL, A. Un test de inteligencia para las organizaciones. Trujillo, Ediciones La Coria, 2003, p.183-192.

NÚÑEZ PAULA, I. (2002). Gestion del Conocimiento. Conceptos, aplicaciones y experiencias. En: Seminario Iberoamericano sobre tendencias modernas en Gerencia de la Ciencia y la Innovacion Tecnologica. IBERGECYT '2002. La Habana: Editorial Academia, p. 185.

OBERCHULSTE (1996). Citado por: PINHEIRO, P. G.; HERNÁNDEZ M. R., and M. L. RAPOSO. La inteligencia organizativa de las empresas del sector textil portugués. 2007 [en línea]. Disponible en Web: <http:// www.dialnet.unirioja.es> [Consulta: 3 de agosto de 2009].

OROZCO S., E. "El lugar de la inteligencia empresarial en el entorno conceptual de la gestión del conocimiento. Evolución en Cuba". El profesional de la información. julioagosto 2001, vol. 10, núm. 7-8, p. 14-22.

OROZCO S., E. Inteligencia empresarial. En: Inteligencia empresarial: Qué y cómo. La Habana: Instituto de Información Científica y Tecnológica, IDICT, 2009, p. 6.

OROZCO, S., E. "Enfoque conceptual de la inteligencia organizacional en algunas fuentes de información. Aplicación en la industria biofarmacéutica". Ciencias de la Información. diciembre, 1998, vol. 29, núm. 4, p.35-45.

PÁEZ URDANETA, I. Gestion de la inteligencia, aprendizaje tecnologico y modernizacion del trabajo informacional. Retos y Oportunidades. Instituto de Estudios del Conocimiento de la Univ. Simon Bolivar/ Consejo Nacional de Investigaciones Cientificas y Tecnologicas. Caracas, 253p.

PINHEIRO, P. G.; HERNÁNDEZ M. R., and M. L. RAPOSO. La inteligencia organizativa de las empresas del sector textil portugués. 2007 [en línea]. Disponible en Web: <http:// www.dialnet.unirioja.es> [Consulta: 3 de agosto de 2009].

RODRÍGUEZ, S. M. and ESCORSA, P. De la información a la inteligencia tecnológica: un avance estratégico. En: Memorias del Seminario Iberoamericano sobre tendencias 
modernas en gerencia de la Ciencia y la Innovación Tecnológica-IBERGECYT '97, GECYT. La Habana, 1997, pp. 297-317.

SHRIVASTA Y GRANT, 1985. Citado por: ESCORSA, P. and MASPONS, R. De la Vigilancia Tecnológica a la Inteligencia Competitiva. Madrid: Pearson Education, 2001, 165p. TAYLOR, R. S. Value-added processes in information systems. Norwood, NJ: Ablex, 1986.

VIZCAYA D. A. Información: procesamiento de contenido. Argentina: Editorial Nuevo Paradigma, 1997, p. 168.

\section{SÍNTESIS CURRICULAR}

Adela Haber Vega. Licenciada en Información Científico-Técnica y Bibliotecología (ICTB) en la Universidad de La Habana, en 1985. Diplomada en Gerencia de Información, en el Centro de Estudios y Desarrollo Profesional en Ciencias de la Información (PROINFO), División del Instituto de Información Científica y Tecnológica (IDICT), en 1999. Ha cursado numerosos cursos de postgrado y participado en eventos nacionales e internacionales, relacionados con su especialidad y con la gestión y gerencia de la ciencia, la tecnología y el medio ambiente.

Anays Más Basnuevo. Lic. en Cibernética Económica, Universidad Estatal "Vladimir Ilich Lenin", Kazán, Rusia, 1988. Doctora en Ciencias de la Información, Facultad de Comunicación, Universidad de La Habana, 2005. Máster en Gerencia de la Ciencia y la Innovación, Instituto de Tecnologías y Ciencias Aplicadas, 2006. Especialista en Inteligencia Empresarial, Instituto de Tecnologías y Ciencias Aplicadas, 2013. Profesor Titular, 2010. Actualmente, trabaja en el Departamento de Servicios de Inteligencia Empresarial de la Consultoría BioMundi/IDICT. Posee 37 publicaciones. Ha recibido e impartido docencia, así como participado en eventos nacionales e internacionales, resultando premiada en algunos de ellos. Tutora de tesis de grado, maestría y doctorado. Es miembro de diferentes comisiones científicas, comités académicos y asociaciones. Sus resultados científicos han recibido el premio de la Academia de Ciencias de Cuba a nivel provincial. El Consejo de Estado de la República de Cuba le otorgó las medallas "Hazaña Laboral” y “Jesús Menéndez”. 
